\title{
THE HELIUM-TO-METALS ENRICHMENT RATIO IN PLANETARY NEBULAE
}

\section{C.M.L. CHIAPPINI and W.J. MACIEL}

Instituto Astronômico e Geofísico da USP, Av. Miguel Stefano 4200, 04301-904 São Paulo SP, Brazil

The helium-to-metals enrichment ratio $(d Y / d Z)$ and the pregalactic helium abundance $\left(Y_{p}\right)$ are important parameters for the understanding of the galactic chemical evolution, and also as a cosmological test to the Big Bang theory. In this work, we present a new determination of these parameters based on a sample of galactic HII regions, HII galaxies, and planetary nebulae.

The determination of $d Y / d Z$ depends mainly on planetary nebulae, while $Y_{p}$ is essentially determined by the low metallicity objects. For the objects with measured abundances of $\mathrm{O}, \mathrm{N}, \mathrm{C}, \mathrm{Ne}, \mathrm{S}, \mathrm{Ar}$, and $\mathrm{Cl}$, the correlation between the total metal abundance $(Z)$ and the abundances of oxygen, nitrogen, and carbon can be studied in detail, so that the slope $d Y / d Z$ can be determined from plots of the helium abundance by mass $Y$ as a function of $Z, \mathrm{O} / \mathrm{H}$ and $\mathrm{N} / \mathrm{H}$. We have taken into account the contamination of the observed abundances of $\mathrm{He}, \mathrm{N}$, and $\mathrm{C}$ in planetary nebulae due to the fresh material produced and dredged up by their central stars.

The results show that $d Y / d Z \geq 5$, which is higher than previously reported. We obtained further evidence that this value depends on the PN type, decreasing for high metallicities. Therefore, the simple linear model of chemical evolution is limited to low metallicities.

Work partially supported by CNPq and FAPESP. 